University of Louisville

ThinkIR: The University of Louisville's Institutional Repository

Faculty Scholarship

3-2017

\title{
Reduced mitochondrial efficiency explains mismatched growth and metabolic rate at supraoptimal temperatures.
}

\author{
Eloy Martinez \\ Virginia Commonwealth University \\ Michael A. Menze \\ University of Louisville \\ Salvatore J. Agosta \\ Virginia Commonwealth University
}

Follow this and additional works at: https://ir.library.louisville.edu/faculty

Part of the Biology Commons

Original Publication Information

Martinez, Eloy, Michael A. Menze, and Salvatore J. Agosta. "Reduced Mitochondrial Efficiency Explains

Mismatched Growth and Metabolic Rate at Supraoptimal Temperatures." 2017. Physiological and

Biochemical Zoology 90(2): 294-298.

This Article is brought to you for free and open access by ThinkIR: The University of Louisville's Institutional Repository. It has been accepted for inclusion in Faculty Scholarship by an authorized administrator of ThinkIR: The University of Louisville's Institutional Repository. For more information, please contact thinkir@louisville.edu. 


\section{Reduced Mitochondrial Efficiency Explains Mismatched Growth and Metabolic Rate at Supraoptimal Temperatures}

\author{
Eloy Martinez ${ }^{1, *}$ \\ Michael A. Menze ${ }^{2}$ \\ Salvatore J. Agosta ${ }^{1,3}$ \\ ${ }^{1}$ Center for Environmental Studies, Virginia Commonwealth \\ University, Richmond, Virginia 23284; ${ }^{2}$ Department of \\ Biology, University of Louisville, Louisville, Kentucky 40292; \\ ${ }^{3}$ Department of Biology, Virginia Commonwealth University, \\ Richmond, Virginia 23284
}

Accepted 10/13/2016; Electronically Published 12/8/2016

\begin{abstract}
The relationship between whole-organism growth and metabolism is generally assumed to be positive and causative; higher metabolic rates support higher growth rates. In Manduca sexta, existing data demonstrate a deviation from this simple prediction: at supraoptimal temperatures for larval growth, metabolic rate keeps increasing while growth rate is decreasing. This mismatch presumably reflects the rising "cost of maintenance" with temperature. Precisely what constitutes this cost is not clear, but we suspect the efficiency with which mitochondria harness oxygen and organic substrates into cellular energy (ATP) is key. We tested this by integrating existing data on $M$. sexta growth and metabolism with new data on mitochondrial bioenergetics across the temperature range $14^{\circ}-$ $42^{\circ} \mathrm{C}$. Across this range, our measure of mitochondrial efficiency closely paralleled larval growth rates. At supraoptimal temperatures for growth, mitochondrial efficiency was reduced, which could explain the mismatch between growth and metabolism observed at the whole-organism level.
\end{abstract}

Keywords: energy balance, temperature, mitochondria, insect, Manduca, OXPHOS, LEAK, energy transduction, cost of maintenance.

*Corresponding author. Present address: Bosque Estatal de Guánica, Departamento de Recursos Naturales y Ambientales, PO Box 366147, San Juan, Puerto Rico 00936; e-mail: martinez.physiology@gmail.com.

Physiological and Biochemical Zoology 90(2):294-298. 2017. (C) 2016 by The University of Chicago. All rights reserved. 1522-2152/2017/9002-6097\$15.00. DOI: $10.1086 / 689871$

\section{Introduction}

Ecological distributions and abundance are largely determined by the ability of organisms to attain positive energy balance (Dunham et al. 1989; Hall et al. 1992). For populations to persist, organisms must take in sufficient energy to pay the "cost of maintenance," with enough energy leftover to do the "work" that fuels somatic and reproductive growth. In ectotherms (i.e., most organisms), energy balance is largely dependent on body temperature, which relies primarily on environmental temperature (Dunham et al. 1989). A central concept in the study of thermal biology is the temperature performance curve (TPC; Huey and Stevens 1979; Angilleta 2009). TPCs for ectotherm growth have been estimated for many species and are characteristically asymmetric (Angilletta 2009). They typically rise gradually from a lower thermal limit to an optimum temperature at which growth rate is maximized and then drop rapidly toward an upper thermal limit. While the link between growth and metabolism is generally assumed to be positive and causative (i.e., higher metabolic rates support higher growth rates), surprisingly little is understood about the precise nature of this relationship (Glazier 2015; Salin et al. 2015).

Work by Kingsolver and Woods (1997) on Manduca sexta (Lepidoptera: Sphingidae) caterpillars illustrates our lack of understanding of the precise nature of the growth-metabolism relationship. Data from this study are replotted in figure $1 a$ and reveal that past the optimum at $34^{\circ} \mathrm{C}$ where growth is decreasing, metabolic rate is still increasing. TPCs for growth and metabolic rate in a second caterpillar species, Lymantria dispar (Lepidoptera: Erebidae), show the same pattern (Agosta et al., unpublished data). Together, these studies indicate a mismatch between growth and metabolism over the temperature range experienced in nature: at supraoptimal temperatures, growth rates decrease while metabolic rates increase. Presumably, this occurs because of the rising energetic cost of sustaining metabolic activity, with decreased energy available for growth. Precisely what constitutes this rising cost of maintenance is not entirely clear but is key to understanding ecological energetics and, therefore, distributions and abundance (Dunham et al. 1989; Hall et al. 1992).

Costs of maintenance in ectotherms are generally attributed to the energetic costs of fueling vital cellular functions (Jobling 1993). In insects with little capacity for anaerobic metabolism (Chamberlin 2004), cellular functions depend almost entirely on ATP supplied by the mitochondrial oxidative phosphorylation (OXPHOS) system. Although the mechanisms of temperature-induced physiological costs are likely multifaceted 
(e.g., ATP-dependent changes in gene expression, upregulation of protein repair mechanisms; Somero 2011), it seems clear that the efficiency with which the mitochondria convert consumed oxygen into ATP plays a major role. This mitochondrial efficiency hypothesis (MEH) rests on two well-established observations: (i) the coupling efficiency between oxygen consumption and ATP production in mitochondria (the respiratory control ratio $[\mathrm{RCR}]$ ) is temperature dependent (Weinstein and Somero 1998; Hardewig et al. 1999; Martinez et al. 2013, 2016) and (ii) non-ATP-producing respiration (LEAK) represents a significant fraction of the cost of maintaining an organism and dramatically increases at supraoptimal temperatures in ectotherms (Hardewig et al. 1999; Abele et al. 2002; Chamberlin 2004; Martinez et al. 2013). Thus, the MEH hypothesizes that reduced mitochondrial energy transduction efficiency, due to increased protonleak respiration, is a key mechanism driving whole-organism performance. This reduction in efficiency therefore may contribute to the observed mismatch between growth and metabolic rates at elevated temperatures seen in $M$. sexta (fig. 1a).

Here we provide an initial test of the hypothesis that mitochondrial efficiency is a key mechanism driving whole-organism growth efficiency. Specifically, we build on the study of $M$. sexta by Kingsolver and Woods (1997) by testing the prediction that thermal reactions norms for mitochondrial efficiency are closely correlated with thermal reaction norms for caterpillar growth and can explain mismatched growth and metabolic rates at supraoptimal temperatures.

\section{Material and Methods}

\section{Chemicals}

All chemicals for respiration measurements were purchased from Sigma-Aldrich (St. Louis, MO) or Fisher Scientific (Fair Lawn, NJ). Water for solution preparation was purified with a Milli-Q Reagent Water System (Billerica, MA) to an electrical resistance of $18 \mathrm{~m} \Omega$.

\section{Study System}

All mitochondrial respiration measurements were conducted with fifth-instar Manduca sexta specimens obtained commercially from Carolina Biological (Burlington, NC). This laboratory strain has been under constant rearing temperature $\left(26.7^{\circ} \mathrm{C}\right)$ and an artificial diet (Carolina Biological). On arrival, all specimens were maintained at $27^{\circ} \mathrm{C}$ and 24 -h illumination and fed an identical diet until further assayed.

\section{Isolation of Mitochondria}

Mitochondria from $M$. sexta caterpillars were obtained by employing a method modified from Harlankar (1986), Keeley (1973), and Martinez et al. (2013). Briefly, caterpillars were dissected, digestive tubes were removed, and the remaining tissue was separated from the cuticle using an ice-cold spatula. Fresh $(\sim 2.0 \mathrm{~g}$ of wet tissue per sample) tissue was minced in an ice-cold petri dish containing $1 \mathrm{~mL}$ of mitochondrial isolation medium (IM;
$250 \mathrm{mM}$ sucrose, $2 \mathrm{mM}$ ethyleneglycol-bis( $\beta$-aminoethyl ether) $N$, $N^{\prime}$-tetraacetic acid [EGTA], $3.4 \mathrm{mM}$ morpholinopropane sulfonic acid [MOPS], $0.5 \%$ fatty acid free bovine serum albumin [BSA], $\mathrm{pH}=7.4,20^{\circ} \mathrm{C}, 293 \mathrm{mmol} \mathrm{kg}^{-1}$ ) and then homogenized in $5 \mathrm{~mL}$ of IM using an ice-cold Dounce homogenizer (Kontes, Vineland, $\mathrm{NJ}$ ) and seven passes with a loose-fitting pestle followed by two passes with a tight-fitting pestle. Homogenate was centrifuged at $650 \mathrm{~g}$ for $10 \mathrm{~min}$ at $4^{\circ} \mathrm{C}$ to remove cellular debris and undisrupted tissue. The supernatant was collected and again centrifuged at $9,600 \mathrm{~g}$ for $15 \mathrm{~min}$ at $4^{\circ} \mathrm{C}$ to sediment the mitochondrial fraction. Pellets were washed with IM, resuspended, and recollected by centrifugation at 9,600 $\mathrm{g}$ for $15 \mathrm{~min}$ at $4^{\circ} \mathrm{C}$ two consecutive times. The final pellet was suspended in $250 \mu \mathrm{L}$ of IM and stored on ice for $1 \mathrm{~h}$ before being assayed.

a

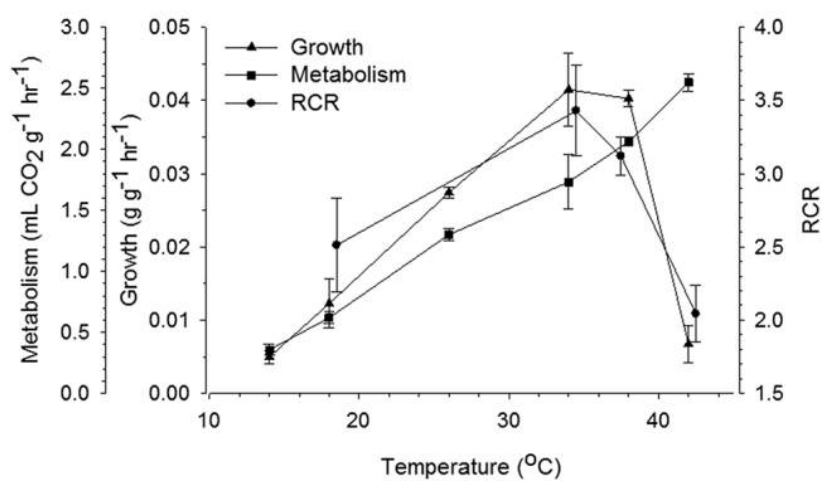

b

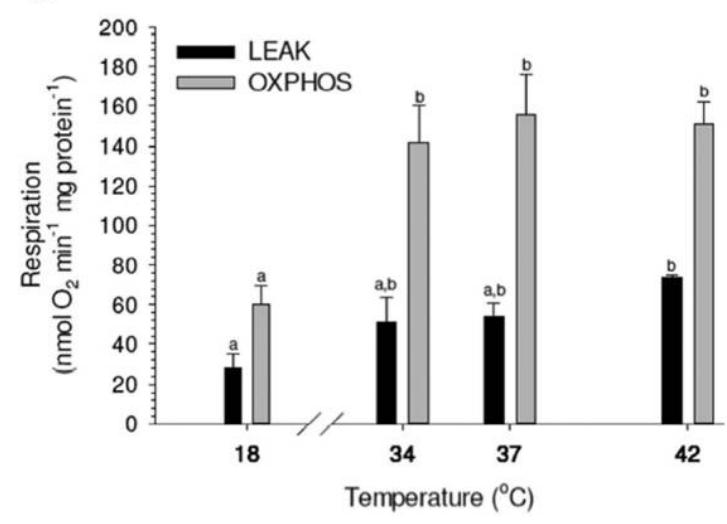

Figure 1. Integrative analysis of the thermal sensitivity of mitochondrial efficiency, metabolic activity, and growth of Manduca sexta caterpillars. $a$, Metabolic activity (squares) and growth (triangles) of fifth-instar $M$. sexta, previously reported by Kingsolver and Woods (1997). Also shown are the mitochondrial respiratory control ratios (RCRs; circles) measured in this study ( $n=3 \pm \mathrm{SEM}$ ). $b$, Mitochondrial leak respiration (LEAK) and oxidative phosphorylation (OXPHOS) as a function of temperature $(n=3 \pm \mathrm{SEM})$. Significant differences within OXPHOS (one-way ANOVA, $P=0.003$ ) and within LEAK (one-way ANOVA, $P=0.014$ ) as a function of temperature are indicated by different letters (Tukey HSD posttests). 
Mitochondrial Respiration

To assess the thermal sensitivity of mitochondria, highresolution respirometry systems were employed. These systems comprise two $2.0-\mathrm{mL}$ water-jacketed respirometric chambers (DW-1, Hansatech Instruments, Norfolk, England) and Clarktype polarographic oxygen electrodes (C-1, Hansatech Instruments). Respirometer chambers were calibrated at the assay temperature in the presence of $500 \mu \mathrm{L}$ of respiration medium (RM) prepared according to Keeley (1973), consisting of $250 \mathrm{mM} \mathrm{su}-$ crose, $0.3 \%$ w/v BSA, $15 \mathrm{mM} \mathrm{KCl,} 5 \mathrm{mM} \mathrm{MgCl}, 0.1 \mathrm{mM}$ EGTA, $25 \mathrm{mM} \mathrm{K}_{2} \mathrm{PO}_{4}$, and $50 \mathrm{mMMOPS}\left(\mathrm{pH}=7.4,20^{\circ} \mathrm{C}, 449 \mathrm{mmol} \mathrm{kg}^{-1}\right)$.

At each measurement temperature $\left(18^{\circ}-42^{\circ} \mathrm{C}\right)$, the background signal was recorded before mitochondrial injection. For each run, $10 \mu \mathrm{L}$ of purified mitochondria $(0.07-0.10 \mathrm{mg}$ of mitochondrial protein) was injected into the respirometer chamber containing $500 \mu \mathrm{L}$ of RM. Electron transport via complexes I, II, and glycerol3-phosphate dehydrogenase (GPDH) to the ETS was assayed concurrently in each run. The respiration not associated with ATP production and broadly defined in this study as oxygen consumption associated with proton conductance, proton slip, and cation cycling at saturating substrate concentrations (LEAK) was initiated by adding $2 \mathrm{mM}$ malate $(\mathrm{M}), 10 \mathrm{mM}$ glutamate $(\mathrm{G})$, $5 \mathrm{mM}$ pyruvate $(\mathrm{P})$, and $10 \mathrm{mM}$ proline, which supplies electrons to complex I via production of NADH by mitochondrial dehydrogenases. Convergent electron entry to the ubiquinone pool via $\mathrm{FADH}_{2}$ was initiated by the addition of $10 \mathrm{mM}$ succinate, and electron entry via GPDH was initiated by adding $10 \mathrm{mM}$ sn-glycerol3-phosphate. To engage OXPHOS, $2 \mathrm{mM}$ ADP was added to the chamber. The proportion of the total respiration coupled to OXPHOS (termed the RCR) was calculated by diving OXPHOS rates by the LEAK rates. Mitochondrial protein was quantified using a Coomassie Plus Reagent Assay (Thermo Scientific, Rockford, IL).

\section{Statistical Analysis}

Significant differences within respiratory states OXPHOS and LEAK as a function of temperature were determined using a one-way ANOVA, in conjunction with Tukey HSD posttests (Sigmaplot 12).

\section{Results and Discussion}

As predicted by the $\mathrm{MEH}$, mitochondrial energy transduction efficiency, calculated here as the RCR, was found to parallel growth rate as a function of temperature in Manduca sexta caterpillars (fig. 1a). As temperature increased from $18^{\circ} \mathrm{C}$ to the previously documented optimal temperature for growth of $34^{\circ} \mathrm{C}$, LEAK rates increased from $27.9 \pm 6.9$ to $50.7 \pm 12.7 \mathrm{nmol} \mathrm{O}_{2}$ $\mathrm{min}^{-1} \mathrm{mg}$ protein ${ }^{-1}$ (fig. 1). In parallel, OXPHOS rates increased from $60.2 \pm 9.4 \mathrm{nmol} \mathrm{O}_{2} \mathrm{~min}^{-1} \mathrm{mg}$ protein ${ }^{-1}$ at $18^{\circ} \mathrm{C}$ to $141.5 \pm$ $19.0 \mathrm{nmol} \mathrm{O}_{2} \mathrm{~min}^{-1} \mathrm{mg}$ protein ${ }^{-1}$ at $34^{\circ} \mathrm{C}$. However, at supraoptimal temperatures between $34^{\circ}$ and $42^{\circ} \mathrm{C}$, OXPHOS rates plateaued while LEAK rates continued to increase from $50.7 \pm$ $12.7 \mathrm{nmol} \mathrm{O}_{2} \min ^{-1} \mathrm{mg}$ protein ${ }^{-1}$ at $34^{\circ} \mathrm{C}$ to $73.6 \pm 1.1 \mathrm{nmol} \mathrm{O}_{2}$ $\min ^{-1} \mathrm{mg}$ protein ${ }^{-1}$ at $42^{\circ} \mathrm{C}$. As a result, RCRs at supraoptimal temperatures decreased (fig. 1b). Nevertheless, despite a rapid increase in LEAK rates at elevated temperatures, mitochondrial respiration coupled to ATP production was observed throughout the temperature range.

Our results combined with the prior results of Kingsolver and Woods (1997) provide a direct link between cellular-level and whole-organism-level energetics and performance in $M$. sexta. The data in figure $1 a$ are consistent with the idea that the "cost of maintenance" across a temperature range is at least partly driven by the efficiency with which the mitochondria convert oxygen consumed by the whole organism into ATP, resulting in decreased biomass accumulation at supraoptimal temperatures despite increased metabolic rates. During life-history stages geared toward the rapid accumulation of biomass (e.g., the larval stages of many species), even small reductions in the efficiency of mitochondrial energy transduction could have large impacts on performance. Scaling up, this thermal dependence of wholeorganism performance on mitochondrial efficiency may play a significant role in determining the dynamics of ecological distributions and abundance, including responses to climate change.

Despite the close similarity between growth and mitochondrial efficiency observed in our study (fig. 1a), there are some caveats with the in vitro approach we took to measure mitochondrial energetics that should be noted. Mitochondrial respiration in vivo is under complex metabolic regulation based on ATP demand and cellular substrate availability. Our in vitro measure of the RCR overestimates the amount of LEAK respiration operative in the animal since coupling efficiency will vary with ATP demand. Therefore, depending on the metabolic constraints of the animal, mitochondrial oxygen consumption will normally occur in an intermediate respiratory state, somewhere between OXPHOS and LEAK rates. Thus, the RCRs we measured in vitro yield an approximation of the worst-case scenario of LEAK-dependent reductions in ATP production efficiency with increasing temperatures in vivo. Nevertheless, our data show that changes in RCRs at temperatures above $35^{\circ} \mathrm{C}$ were driven by an increase in LEAK respiration and not by reductions in the OXPHOS activity. This increase in proton conductance of the inner mitochondrial membrane at elevated temperatures reduces RCR values, decreases cellular ATP yield per substrate consumed, and may negatively impact growth performance.

Our results are in close agreement with previous studies on the thermal performance of invertebrate mitochondria (Abele et al. 2002; Sokolova and Sokolov 2005; Cottin et al. 2012). These studies confirm that proton leak is a temperature-sensitive process by which the proton motive force is dissipated to sustain a relatively stable membrane potential $(\Delta \psi)$. Furthermore, as expected for mitochondria under phosphorylating conditions, Chamberlin (2004) found a lower membrane potential under OXPHOS conditions compared to LEAK respiration in $M$. sexta but little to no changes in the membrane potentials with changes in temperature. In the investigated temperature range $\left(15^{\circ}-35^{\circ} \mathrm{C}\right)$, the mitochondrial membrane potential for nonphosphorylating mitochondria remained close to $200 \mathrm{mV}$, while LEAK respiration increased about sixfold. This evidence further suggests that proton cycling may regulate membrane potential at supraoptimal tem- 


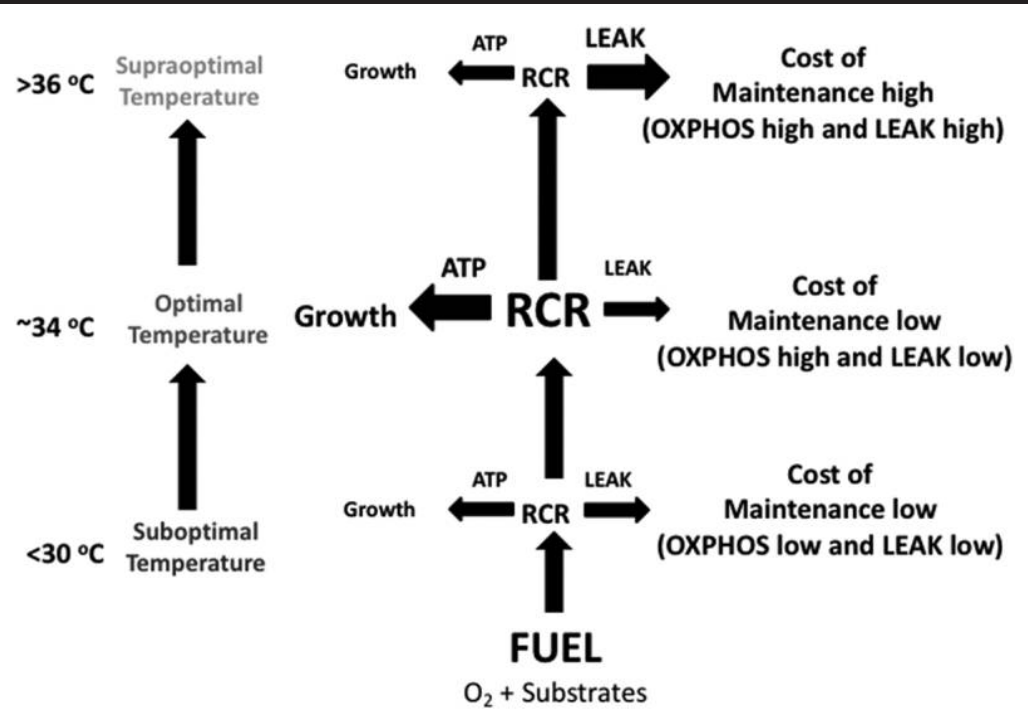

Figure 2. Conceptual framework of the mitochondrial efficiency hypothesis to explain mismatched growth and metabolism at supraoptimal temperatures. Temperature-induced metabolic costs of maintenance in ectotherms are attributed to the energetic costs of fueling vital cellular functions, all of which depend on ATP mostly supplied by mitochondria. However, little attention has been directed toward the thermal sensitivity of the energy transduction efficiency of mitochondria, which dictates the proportion of carbon substrates and oxygen necessary to sustain a fixed ATP supply, via the oxidative phosphorylation (OXPHOS) system. At supraoptimal temperatures, the respiratory control ratio (RCR) is reduced due to an increase in mitochondrial leak respiration (LEAK), which contributes to the rising cost of maintenance. This concept is illustrated by a shift in the sizes of arrows and text at supraoptimal temperatures. See text for further details. A color version of this figure is available online.

peratures and that proton leak likely acts as a safety valve that reduces reactive oxygen species formation and diminishes the risks of oxidative stress (Brand 2000). Independent of the mechanisms by which LEAK respiration is increased at elevated temperatures, proton leak will utilize substrates without generating ATP, which otherwise could be allocated to somatic and reproductive growth (fig. 2). Furthermore, food utilization, measured as methionine uptake plateaus and mass-specific consumption rates, decreases above $38^{\circ} \mathrm{C}$ in $M$. sexta (Kingsolver and Woods 1997). These effects may operate in parallel with the reduction in RCR and further reduce the growth rate of $M$. sexta caterpillars at high temperatures.

Finally, we realize that the concept of aerobic limitations on whole-organism performance at high temperatures is not novel. The model of oxygen- and capacity-limited thermal tolerance (OCLTT) advanced by Pörtner and colleagues (Pörtner 2002, 2010; Pörtner et al. 2005) posits that a mismatch between oxygen delivery and demand limits aerobic scope at supraoptimal temperatures, which reduces overall performance. As body temperatures rise to the high end of the physiological range, oxygen demand by cells becomes greater than the organisms' ability to supply it. To compensate for reduced aerobic scope, molecular adjustments are needed, including an increased anaerobic metabolism. Experiments appear to support the OCLTT as a general set of mechanisms behind sensitivities to high temperatures in marine ectotherms (reviewed in Verberk et al. 2016), which routinely operate in relatively lowoxygen environments (i.e., water). However, in air-breathing insects, there is little evidence that the OCLTT applies under normoxia (reviewed in Verberk et al. 2016). Alternatively, or in addition, our results suggest that the thermal sensitivity of mitochondrial energy transduction efficiency plays a major role in limiting organismal thermal performance (fig. 2), especially in life stages whose primary function is growth (e.g., caterpillars). Although the evidence presented here is limited to a laboratory strain of a single species, we suspect the model in figure 2 is general and applies to many other species. We have already observed a mismatch between growth rate and metabolic rate at supraoptimal temperatures in another caterpillar species (Lymantria dispar; Agosta et al., unpublished data) and are currently testing the hypothesis that it can be explained, at least in part, by mitochondrial efficiency.

\section{Acknowledgments}

We wish to acknowledge the excellent article by Kingsolver and Woods (1997) used to recalculate metabolic activity and growth of fifth-instar Manduca sexta caterpillars. Our work was partially supported by a Presidential Research Quest Fund to S.J.A. from Virginia Commonwealth University. We have no competing interests that might have influenced the work described in this manuscript. E.M., M.A.M., and S.J.A. conceived and designed the experiments. E.M. performed the experiments. E.M., M.A.M., and S.J.A. analyzed the data and prepared the manuscript.

\section{Literature Cited}

Abele D., K. Heise, H.O. Pörtner, and S. Puntarulo. 2002.

Temperature dependence of mitochondrial function and 
production of reactive oxygen species in the intertidal mud clam Mya arenaria. LExp Biol 205:1831-1841.

Angilletta M.J. 2009. Thermal adaptation: a theoretical and empirical synthesis. Oxford University Press, Oxford.

Brand M. 2000. Uncoupling to survive? the role of mitochondrial inefficiency in ageing. Exp Gerentol 35:811-820.

Chamberlin M.E. 2004. Top-down control analysis of the effect of temperature on ectotherm oxidative phosphorylation. Am J Physiol 287:R794-R800.

Cottin D., D. Roussel, N. Foucreau, F. Hervant, and C. Piscart. 2012. Disentangling the effects of local and regional factors on the thermal tolerance of freshwater crustaceans. Naturwissenschaften 99:259-264.

Dunham A.E., B.W. Grant, and K.L. Overall. 1989. Interfaces between biophysical and physiological ecology and the population ecology of terrestrial vertebrate ectotherms. Physiol Zool 62:335-355.

Glazier D.S. 2015. Is metabolic rate a universal "pacemaker" for biological processes? Biol Rev 90:377-407.

Halarnkar P.P., C.R. Heisler, and G.J. Blomquist. 1986. Propionate catabolism in the housefly Musca domestica and the termite Zootermopsis nevadensis. Insect Biochem 16:455-461.

Hall C.A., J.A. Stanford, and F.R. Hauer. 1992. The distribution and abundance of organisms as a consequence of energy balances along multiple environmental gradients. Oikos 65:377-390.

Hardewig I., H. Pörtner, and L. Peck. 1999. Thermal sensitivity of mitochondrial function in the Antarctic notothenioid Lepidonotothen nudifrons. IComp Physiol B 169:597-604.

Huey R.B. and R. Stevenson. 1979. Integrating thermal physiology and ecology of ectotherms: a discussion of approaches. Am Zool 19:357-366.

Jobling M. 1993. Bioenergetics: feed intake and energy partitioning. Pp. 1-44 in J.C. Rankin and F. Jensen, eds. Fish ecophysiology. Chapman \& Hall, London.

Keeley L.L. 1973. Characterization of insect fat body mitochondria isolated by a rapid procedure. J Comp Physiol B 46:147-151.
Kingsolver J.G. and H.A. Woods. 1997. Thermal sensitivity of growth and feeding in Manduca sexta caterpillars. Physiol Biochem Zool 70:631-638.

Martinez E., E. Hendricks, M.A. Menze, and J.J. Torres. 2016. Physiological performance of warm-adapted marine ectotherms: thermal limits of mitochondrial energy transduction efficiency. J Comp Physiol B 191:216-225.

Martinez E., M. Menze, and J. Torres. 2013. Mitochondrial energetics of benthic and pelagic Antarctic teleosts. Mar Biol 160:2813-2823.

Pörtner H.O. 2002. Climate variations and the physiological basis of temperature dependent biogeography: systemic to molecular hierarchy of thermal tolerance in animals. J Comp Physiol A 132:739-761.

- 2010. Oxygen- and capacity-limitation of thermal tolerance: a matrix for integrating climate-related stressor effects in marine ecosystems. LExp Biol 213:881-893.

Pörtner H.O., D. Storch, and O. Heilmayer. 2005. Constraints and trade-offs in climate-dependent adaptation: energy budgets and growth in a latitudinal cline. Sci Mar 69:271-285.

Salin K., S.K. Auer, B. Rey, C. Selman, and N.B. Metcalfe. 2015. Variation in the link between oxygen consumption and ATP production, and its relevance for animal performance. Proc R Soc B 282:20151028.

Sokolova I. and E. Sokolov. 2005. Evolution of mitochondrial uncoupling proteins: novel invertebrate UCP homologues suggest early evolutionary divergence of the UCP family. FEBS Lett 579:313-317.

Somero G.N. 2011. Comparative physiology: a "crystal ball” for predicting consequences of global change. Am I Physiol 301: $\mathrm{R} 1-\mathrm{R} 14$.

Verberk W.C., J. Overgaard, R. Ern, M. Bayley, T. Wang, L. Boardman, and J.S. Terblanche. 2016. Does oxygen limit thermal tolerance in arthropods? a critical review of current evidence. J Comp Physiol A 192:64-78.

Weinstein R. and G. Somero. 1998. Effects of temperature on mitochondrial function in the Antarctic fish Trematomus bernacchii. L Comp Physiol B 168:190-196. 